\title{
Submillimeter imaging spectroscopy of the Horsehead nebula ${ }^{\star}$
}

\author{
S. D. Philipp ${ }^{1}$, D. C. Lis ${ }^{2}$, R. Güsten ${ }^{1}$, C. Kasemann ${ }^{1}$, T. Klein ${ }^{1}$, and T. G. Phillips ${ }^{2}$ \\ 1 Max-Planck-Institut für Radioastronomie, Auf dem Hügel 69, 53121 Bonn, Germany \\ e-mail: sphilipp@mpifr-bonn.mpg.de \\ 2 California Institute of Technology, Downs Laboratory of Physics 320-47, Pasadena, CA 91125, USA
}

Received 30 May 2005 / Accepted 16 March 2006

\section{ABSTRACT}

\begin{abstract}
We present $\sim 15$ arcsecond resolution single-dish imaging of the Horsehead nebula in the CI (1-0) and CO (4-3) lines, carried out using the CHAMP array at the Caltech Submillimeter Observatory (CSO). The data are used together with supporting observations of the (2-1) transitions of the CO isotopologues to determine the physical conditions in the atomic and molecular gas via Photon Dominated Region (PDR) modeling. The CO (4-3)/(2-1) line ratio, which is an excellent tracer of the direction of the incoming UV photons, increases at the western and northern edges of the nebula, confirming that the illumination is provided mostly by the stars $\sigma$ and $\zeta$ Orionis. The observed line intensities are consistent with PDR models with an $\mathrm{H}$ nuclei volume density of $\sim 3-7 \times 10^{4} \mathrm{~cm}^{-3}$. The models predict a kinetic temperature of $\sim 12 \mathrm{~K}$ and a $\mathrm{C}^{18} \mathrm{O}$ fractional abundance with respect to $\mathrm{H}$ atoms of $2.4 \times 10^{-7}$ in the shielded region, which in turn imply a total molecular mass of $\sim 24 M_{\odot}$ in the $\mathrm{C}^{18} \mathrm{O}$ filament. The outer halo, devoid of $\mathrm{C}^{18} \mathrm{O}$, but traced by the CI emission has a comparable density and contributes additional $\sim 13 M_{\odot}$ of material, resulting in an upper limit of $\sim 37 M_{\odot}$ for the total molecular mass of the nebula.
\end{abstract}

Key words. ISM: individual: objects: Horsehead nebula - ISM: molecules - ISM: structure - ISM: abundances - ISM: clouds stars: formation

\section{Introduction}

PDRs are surface layers of interstellar molecular clouds exposed to intense fluxes of FUV photons from external sources, e.g. nearby hot $\mathrm{OB}$ stars or clusters. These regions are strong sources of far-infrared atomic fine-structure line emission of singly ionized carbon (CII) and neutral oxygen (OI), molecular hydrogen $\left(\mathrm{H}_{2}\right)$ ro-vibrational transitions, as well as infrared continuum emission. Deeper into the transition region, at $A_{\mathrm{v}} \sim 1-3 \mathrm{mag}$ of visual extinction, as the FUV flux is gradually attenuated by the intervening dust, strong emission of fine-structure lines of neutral atomic carbon $(\mathrm{CI})$ appears, along with carbon monoxide $(\mathrm{CO})$ rotational lines. The $\mathrm{CO}$ emission defines the regions referred to as molecular clouds, which harbor all known sites of ongoing star formation in the Milky Way. In recent years, PDRs have been subjects of numerous observational studies, as well as theoretical modeling aimed at deriving the physical conditions and chemical abundance profiles of the atomic and molecular species as a function of visual extinction (see Hollenbach \& Tielens 1997, 1999, for recent reviews).

The Horsehead, best known from its stunning optical images, is located in the HII region IC 434 in Orion. It is a dense clump of molecular material at the periphery of the L1630 molecular cloud, protruding into the HII region and illuminated by the FUV radiation from $\sigma$ Orionis, an $\mathrm{O} 9.5 \mathrm{~V}$ star located at a projected distance of $\sim 4 \mathrm{pc}$. The region has been a subject of several recent and ongoing studies at millimeter wavelengths. Pound et al. (2003) presented high-angular resolution CO (1-0) observations of the region and found that the $\mathrm{CO}$ emission closely matches

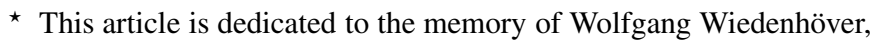
who lead the efforts on the developments of the correlator array backend MACS for CHAMP. the outline of the dark patch seen at visual wavelengths. They also showed that the molecular cloud exhibits a pronounced velocity gradient of $5 \mathrm{~km} \mathrm{~s}^{-1} \mathrm{pc}^{-1}$ from the NE to the $\mathrm{SW}$ and derived a total mass of the molecular material of $27 M_{\odot}$. The presence of several near-infrared and IRAS sources in the vicinity of the Horsehead (Reipurth \& Bouchet 1984; Sandell et al. 1985) provides direct evidence for recent star formation in the region. Yamauchi et al. (2000) report a detection of a faint X-ray point source using ASCA with a luminosity of $6 \times 10^{30} \mathrm{erg} \mathrm{s}^{-1}$ $(0.5-10 \mathrm{keV})$, which are also interpreted to be young stellar objects.

Zhou et al. (1993) derive a mean $\mathrm{H}$ nuclei density in the Horsehead nebula of $\sim 2 \times 10^{4} \mathrm{~cm}^{-3}$ and an incident UV flux of 20-100 Habing units (Habing 1968) based on their observations of CO, ${ }^{13} \mathrm{CO}$, CS, and CII emission. Kramer et al. (1996) derive a similar density of $3 \times 10^{4} \mathrm{~cm}^{-3}$ from various $\mathrm{CO}$ line ratios. The Horsehead can be identified with the CS (2-1) clump 42 in the survey of Lada et al. (1991), who derive a virial mass of $35 M_{\odot}$ and an effective radius of $0.17 \mathrm{pc}$, leading to an $\mathrm{H}$ nuclei density of $7 \times 10^{4} \mathrm{~cm}^{-3}$, if the cloud is in virial equilibrium. Mid-infrared emission from the Horsehead (Abergel et al. 2002, 2003; also the recent analysis of Habart et al. 2005, published after this manuscript has been submitted) reveals a sharp filament at the illuminated edge of the nebula. A comprehensive study of small carbon chains and rings in this region was presented by Teyssier et al. (2004) and Pety et al. (2005).

Here we present high-angular resolution single-dish observations of the Horsehead in the CI (1-0) and CO (4-3) emission, together with supporting observations of the (2-1) transitions of the CO isotopologues. The data are used to derive the physical conditions in the molecular gas through PDR modeling. 


\section{Observations}

The submm observations discussed here were performed using CHAMP at the CSO in two observing periods in January and September 2001 . The complementary $\mathrm{C}^{18} \mathrm{O}(2-1)(219.56 \mathrm{GHz})$ and $\mathrm{CO}(2-1)(230.54 \mathrm{GHz})$ data were obtained at the IRAM $30 \mathrm{~m}$ with the Heterodyn Receiver Array HERA (Schuster et al. 2002) in May 2003.

CHAMP, the "Carbon Heterodyne Array of the MPIfR" was operated at the CSO until June 2003. The CHAMP array consists of $2 \times 8$ elements, with $\sqrt{2 \theta_{\mathrm{mb}}}$ spacing of the pixels on the sky, where $\theta_{\mathrm{mb}}$ is the FWHM beam size (see, e.g., Güsten et al. 1998, for a design description). The tuning range of the receiver covered the $625 \mu \mathrm{m}$ atmospheric window ( $\sim 450-495 \mathrm{GHz}$, limited by the local oscillator), including the $J=4-3$ transition of excited warm $\mathrm{CO}(461.06 \mathrm{GHz})$ as well as the fine-structure transition of atomic carbon [CI] $(492.16 \mathrm{GHz})$. Cold optics and a Martin-Puplett interferometer serving as a single-sideband filter, that terminated the image band on a $15 \mathrm{~K}$ absorber, allowed for SSB-system temperatures well below $1000 \mathrm{~K}$ in good atmospheric conditions. The frontend was linked to a flexible autocorrelator backend, with a maximum bandwidth of $2 \mathrm{GHz}$ (2048 channels) per frontend pixel. For the Horsehead observations we operated the spectrometer in one of its higher resolution mode, providing a resolution of $0.15 \mathrm{~km} \mathrm{~s}^{-1}$ per channel. A network of fast Digital Signal Processors with optimized Fast Fourier Transform algorithms processed the correlator raw data realtime.

The instrument control could handle total power, frequency and wobble switch observations in pointing, raster and on-thefly-modes, allowing to adopt the optimal observing strategy for a given source. The Horsehead observations were performed in frequency-switched (at $1 \mathrm{~Hz}$ switch frequency) onthe-fly scanning mode. The symmetric switch throw was set to $\pm 15-25 \mathrm{MHz}$ and the mapping was performed both in RA and Dec directions with a sampling of $8^{\prime \prime}$. The array was kept in its orientation with respect to the sky using the de-rotator of the cryostat.

Pointing was established by scans across the planets to an accuracy of a few arcsec rms, the CHAMP beams were diffraction limited (15" at $464 \mathrm{GHz}$ and $14.5^{\prime \prime}$ at $492 \mathrm{GHz}$ ). Calibration of the antenna temperature scale was performed by the standard chopper wheel method, coupling efficiencies were determined by flux measurements of the planets. The efficiencies determined on Jupiter and Mars were $\left\langle\eta_{\mathrm{c}}\right\rangle=0.56$ and 0.45 , respectively, with max $10 \%$ variation between the pixels. The actual zenith sky opacity during observations was between 0.06 and 0.11 at $225 \mathrm{GHz}$.

The complementary observations of the $\mathrm{C}^{18} \mathrm{O}(2-1)$ and CO (2-1) lines with the HEterodyne Receiver Array (HERA) on the IRAM $30 \mathrm{~m}$ telescope on Pico Veleta, Spain were obtained in total power on-the-fly scanning mode, again with a sampling of $8^{\prime \prime}$ and mapping performed in both, RA and Dec directions. The array was inclined $18.5^{\circ}$ to the equatorial frame using HERA's derotation system. For details of the set-up and instrument description we refer to Schuster et al. (2002). Pointing was established on Saturn and the nearby quasar 0420-014, for calibration we assumed a main-beam coupling efficiency of 0.52 . We estimate the calibration uncertainty to be $\sim 20 \%$. The observations were performed under average to good weather conditions with zenith opacities of $\tau \sim 0.16-0.30$.

In addition, supporting observations of the ${ }^{13} \mathrm{CO}(2-1)$ $(220.40 \mathrm{GHz})$ transition, with 31" angular resolution, were carried out, as a bad weather backup observing program, using

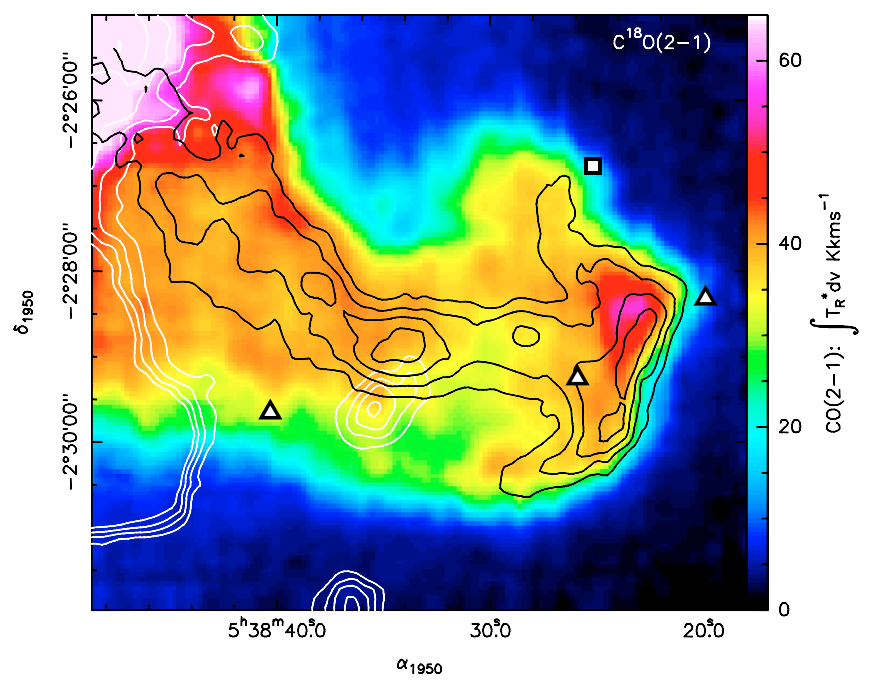

Fig. 1. Distribution of the CO (2-1) integrated intensity, convolved to $14^{\prime \prime}$ resolution, in the Horsehead in the $8.2-11.7 \mathrm{~km} \mathrm{~s}^{-1}$ and $11.7-13.8 \mathrm{~km} \mathrm{~s}^{-1}$ velocity intervals (color-scale image and white contours, respectively). The low-velocity interval includes most of the emission from the Horsehead. The $\mathrm{C}^{18} \mathrm{O}(2-1)$ emission (black contours, 9.2-11.7 $\mathrm{km} \mathrm{s}^{-1}$ velocity interval, also convolved to $14^{\prime \prime}$ resolution) reveals a narrow filament running through the center of the head, referred to as the neck, and a bright hammer-like structure on the top of the head. A compact high-velocity CO clump, most likely unrelated to the head is located $\sim 45^{\prime \prime}$ south of the main $\mathrm{C}^{18} \mathrm{O}$ peak in the neck. Contour levels for $\mathrm{CO}$ are 10 to $25 \mathrm{~K} \mathrm{~km} \mathrm{~s}^{-1}$ with an interval of $5 \mathrm{~km} \mathrm{~s}^{-1}$ and for $\mathrm{C}^{18} \mathrm{O} 2.5$ to $7 \mathrm{~K} \mathrm{~km} \mathrm{~s}^{-1}$ with an interval of $1.5 \mathrm{~K} \mathrm{~km} \mathrm{~s}^{-1}$. Triangles mark the locations of IRAS sources (Pound et al. 2003).

the CSO facility receiver and the CHAMP autocorrelator backend.

\section{Morphology of the Horsehead nebula}

Figure 1 shows the overall morphology of the Horsehead nebula as traced by the $\mathrm{CO}(2-1)$ and $\mathrm{C}^{18} \mathrm{O}(2-1)$ emission observed with HERA with $11^{\prime \prime}$ angular resolution, smoothed to $16^{\prime \prime}$. Figure 2 shows the $\mathrm{CO}$ (4-3) emission overlaid on the corresponding mosaic of the NOAO Star Formation Survey Data 1997 by $\mathrm{J}$. Bally (private communication) in $\mathrm{H} \alpha$ emission.

The CO data, both (2-1) and (4-3), show the characteristic outline of the nebula as seen in the optical images of the region, while the $\mathrm{C}^{18} \mathrm{O}$ emission is confined to a narrow filament running through the center of the head (black contours in Fig. 1; hereafter referred to as the neck). In addition, a bright, hammer-like $\mathrm{C}^{18} \mathrm{O}$ structure is seen near the top of the head. The $\mathrm{CO}$ emission associated with the Horsehead is confined to velocities in the range $8.2-11.7 \mathrm{~km} \mathrm{~s}^{-1}$ (color image, Fig. 1). Two compact CO clumps are seen at higher velocities $\left(11.7-14 \mathrm{~km} \mathrm{~s}^{-1}\right)$, one of them just $\sim 45^{\prime \prime}$ south of the brightest $\mathrm{C}^{18} \mathrm{O}$ peak in the neck (white contours in Fig. 1). This is either a background or a foreground source, kinematically distinct from the head. No $\mathrm{C}^{18} \mathrm{O}$ or ${ }^{13} \mathrm{CO}$ for which we observed some snapshots using the CSO, emission in this velocity range has been detected. Therefore, they appear to be a relatively low column density condensation (see Sect. 6).

Figure 3 shows the distribution of the $\mathrm{CI}(1-0)$ and $\mathrm{CO}(4-3)$ emission observed with CHAMP at $\sim 20$ and 16" angular resolution (color image and black contours, respectively). The $\mathrm{CO}$ (4-3) emission is quite uniform, with the exception of a bright patch near the top of the head. The CI emission is much less enhanced in this region, compared to $\mathrm{CO}(4-3)$, and the 


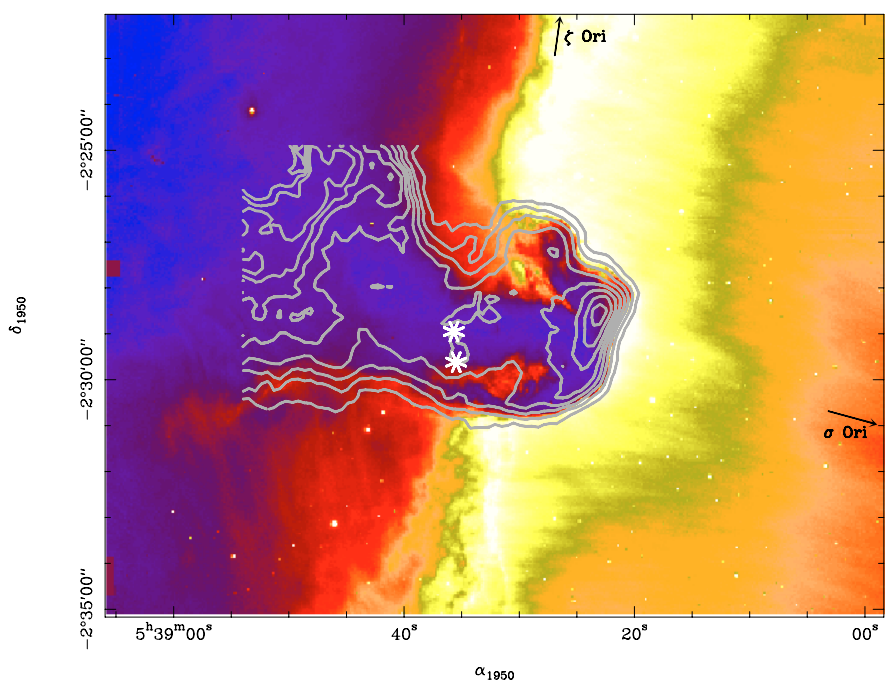

Fig. 2. Distribution of the $\mathrm{CO}(4-3)$ integrated intensity in the Horsehead in the $8.9-11.7 \mathrm{~km} \mathrm{~s}^{-1}$ velocity interval (contours as in Fig. 1) overlaid on the corresponding mosaic of the NOAO Star Formation Survey Data 1997 by J. Bally (private communication) in $\mathrm{H} \alpha$ emission. The $\mathrm{CO}$ emission traces the characteristic outline of the nebula as seen in the optical images of the region. The directions towards the illuminating stars $\sigma$ and $\zeta$ Orionis are indicated by the arrows and the positions, at which the sample spectra were extracted, as shown in Fig. 4, are marked by white stars.

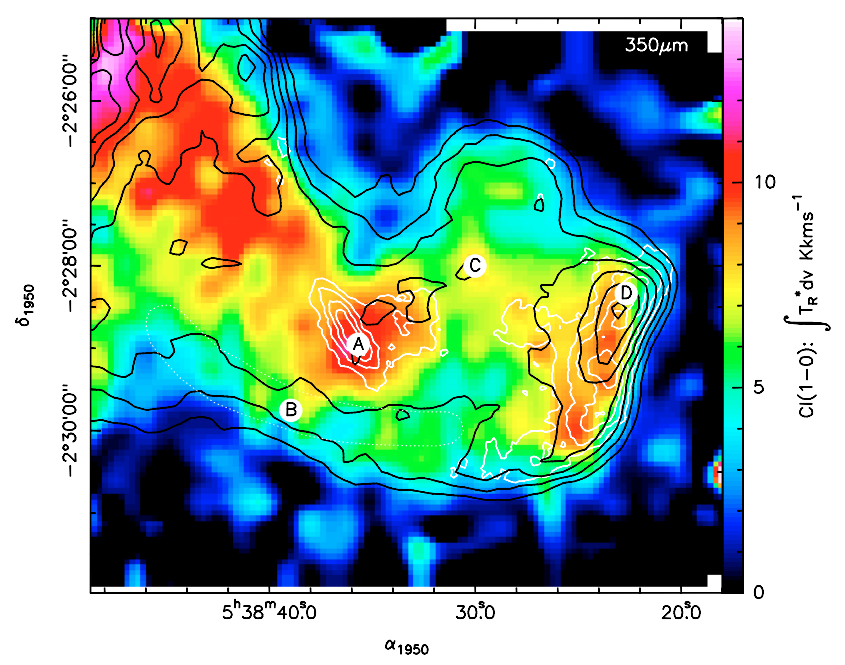

Fig. 3. Distribution of the CI integrated intensity in the $8.9-11.7 \mathrm{~km} \mathrm{~s}^{-1}$ velocity interval (color-scale) and the CO (4-3) emission (black contours). White contours outline the distribution of the $350 \mu \mathrm{m}$ continuum emission (from D. Dowell, private communication). White circles and the dotted polygon mark the regions selected for detailed PDR modeling. Contour levels for $\mathrm{CO}$ are 10 to $40 \mathrm{~K} \mathrm{~km} \mathrm{~s}^{-1}$ with an interval of $5 \mathrm{~K} \mathrm{~km} \mathrm{~s}^{-1}$ and for the dust continuum 0.5 to $2 \mathrm{Jy}$ beam $^{-1}$ with an interval of $0.35 \mathrm{Jy} \mathrm{beam}^{-1}$. The CI image has been convolved to $20^{\prime \prime}$ angular resolution to improve the signal-to-noise ratio.

brightest CI peak is found instead in the middle of the neck, at the SW end of a compact $350 \mu \mathrm{m}$ continuum source (white contours; D. Dowell, private communication). The white circles and the dotted polygon in Fig. 3 mark the location of regions selected for detailed PDR modeling (Sect. 4). The distribution of the CI (1-0) emission is strikingly different from that of the CII $158 \mu \mathrm{m}$ emission, as imaged by Zhou et al. (1993) with the FIFI instrument aboard the Kuiper Airborne Observatory, with a beam size of each detector of $55^{\prime \prime}$ in the $5 \times 5$ array. The velocityintegrated CII emission (Fig. 2 of Zhou et al. 1993) shows two

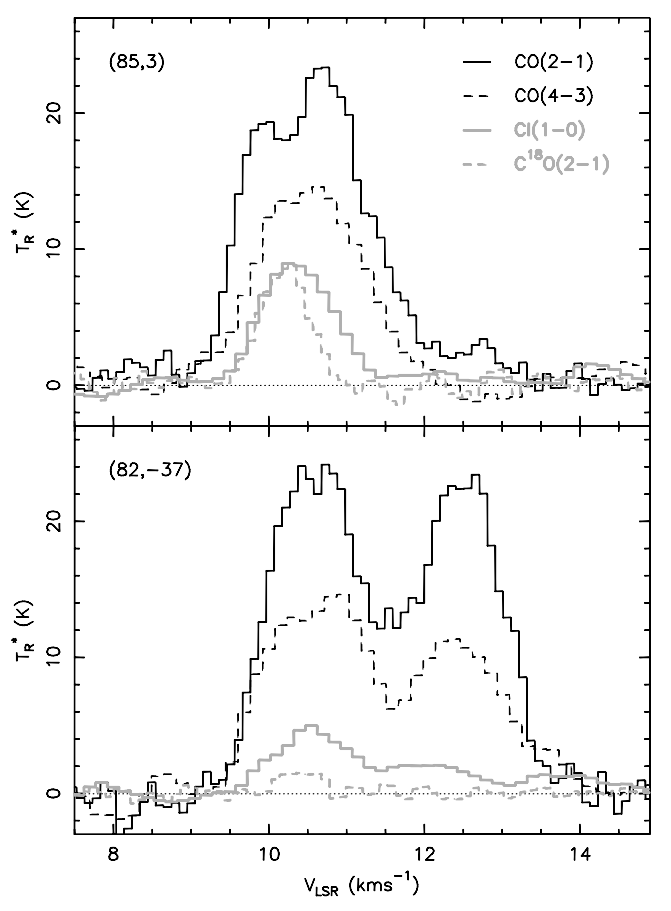

Fig. 4. Sample spectra at the $(85,3)$ and $(82,-37)$ positions (position A and the high velocity clump, respectively).

peaks north and south of the CI peak in the neck and an east-west filament just north of the $\mathrm{C}^{18} \mathrm{O}$ neck. These differences may be partially attributed to the low spectral resolution of the CII data, which does not allow for separating emission associated with the Horsehead from the bright high-velocity emission from the edge of the L1630 molecular cloud (white contours in Fig. 1; see also Fig. 1 of Pound et al. 2003).

Figure 4 shows example spectra towards the CI peak and the high-velocity clump positions, with offsets of $(85,3)$ and $(82,-37)$ arcsec, respectively, with respect to our nominal reference position $\left.\alpha_{1950}=5^{\mathrm{h}} 38^{\mathrm{m}} 30^{\mathrm{s}}, \delta_{1950}=-2^{\circ} 29^{\prime} 00^{\prime \prime}\right)$. The excitation of the high-velocity gas is different from the Horsehead nebula gas proper and the two are likely physically not associated. Figure 5 displays the $\mathrm{CO}(2-1)$ and $\mathrm{C}^{18} \mathrm{O}(2-1)$ velocity channel maps. We confirm the pronounced velocity gradient along the neck, previously seen by Pound et al. (2003; $5 \mathrm{~km} / \mathrm{s} / \mathrm{pc}$ ), with the horse's nose being most redshifted.

\section{PDR modeling of the Horsehead}

The CI and CO isotopologue emission from the Horsehead nebula have been modeled using the Meudon PDR code (Abgrall et al. 1992; Le Bourlot et al. 1993; Le Bourlot 2000 and more recently Pety et al. 2005). This model treats the PDR as a onedimensional plane-parallel slab (similar to the one shown in Fig. 1 of Sternberg 2005), with hydrogen density $n_{\mathrm{H}}$ and an incident radiation field $G_{0}$ (in Draine units, $2.7 \times 10^{-3} \mathrm{erg} / \mathrm{s} / \mathrm{cm}^{2}$; one Draine unit equals 1.7 Habing units). Most of the models presented here assume a constant density. A comparison with isobaric models is presented in Sect. 4.2. The chemical network uses 450 chemical species and roughly 5000 reactions. "Standard" abundances used for the most abundant elements are are $\mathrm{C} / \mathrm{H}=1.38 \times 10^{-4}, \mathrm{O} / \mathrm{H}=3.02 \times 10^{-4}, \mathrm{~N} / \mathrm{H}=7.95 \times 10^{-5}$ with isotopic abundances ${ }^{13} \mathrm{C} / \mathrm{H}=1.6 \times 10^{-6},{ }^{18} \mathrm{O} / \mathrm{H}=6 \times 10^{-7}$. The photodissociation of the $\mathrm{CO}$ isotopomeres is treated in details. For a detailed description of the code, we refer the interested reader to Le Petit et al. (2006). 


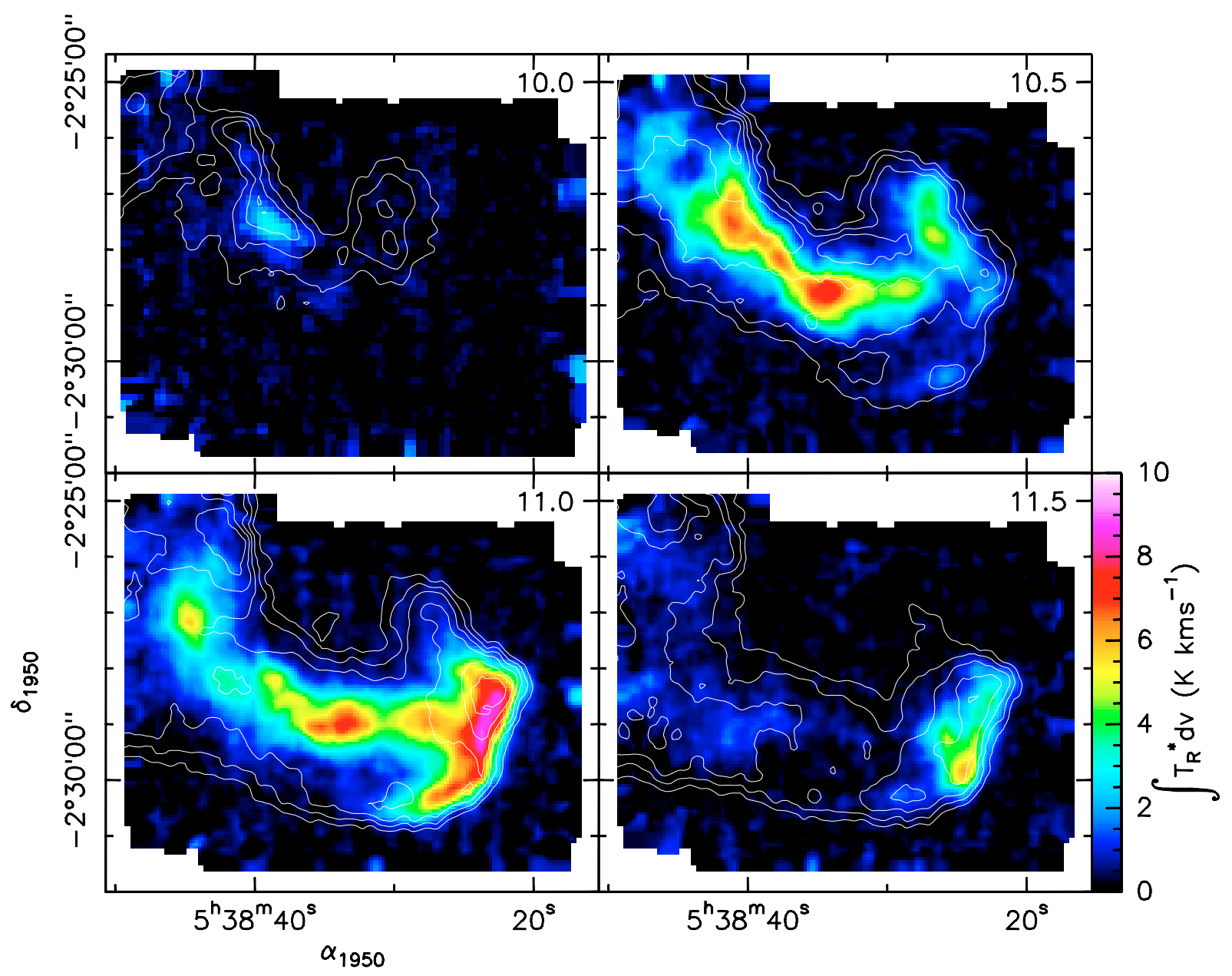

Fig. 5. Velocity channel maps of the $\mathrm{C}^{18} \mathrm{O}(2-1)$ and $\mathrm{CO}(2-1)$ emission (color image and white contours, respectively). Contour levels are 5 to $20 \mathrm{~K}$, with an interval of $5 \mathrm{~K}$. The center velocity of each channel is shown in the upper right corner, channel width $0.5 \mathrm{~km} \mathrm{~s}^{-1}$.

In order to characterize the physical conditions in the neck, away from the narrow, limb-brightened filament on the top of the head, we have selected three positions. Position A corresponds to the location of the CI peak in the neck, at the SW end of the $350 \mu \mathrm{m}$ continuum source. Position B represents the average envelope emission, $\sim 70^{\prime \prime}$ south of the $\mathrm{C}^{18} \mathrm{O}$ filament (see the dotted polygon in Fig. 3); this region is characterized by relatively uniform emission in all the tracers studied here. Finally, position C corresponds to a $\mathrm{CO}$ and $\mathrm{CI}$ peak in the "mouth" region. We assume that the cloud geometry is that of a roughly cylindrical filament attached to the main molecular cloud at the bottom of the neck. The FUV illumination is provided primarily by $\sigma$ Orionis (equivalent $G_{0} \simeq 60$, e.g. Pety et al. 2005). The top of the head is directly exposed to the stellar radiation, while the neck is illuminated indirectly by the radiation scattered and reprocessed by the dust. In the models for the neck area (positions A-C), we assume double-sided illumination with the same FUV intensity on the front and back side of the cloud, characterized by an enhancement factor $G_{0}$ over Draine's value. The only parameters adjusted in the models are: $G_{0}$, the hydrogen volume density, $n_{\mathrm{H}}$ (assumed to be uniform in our constant density models) or the gas pressure (in our isobaric models, Sect. 4.2), and the total visual extinction through the cloud, $A_{\mathrm{V}}$. The dust extinction curve toward $\sigma$ Ori has not been measured. We thus use the dust extinction curve parameters derived by Fitzpatrick \& Massa (1990) toward HD 37903, the star illuminating the nearby NGC 2023 reflection nebula. The HD 37903 extinction curve is relatively similar to the standard Galactic extinction curve (Seaton 1979).

\subsection{Constant density models}

The model parameters are listed in Table 1. The integrated line intensities, computed assuming a face-on geometry and a turbulent velocity dispersion of $0.8 \mathrm{~km} \mathrm{~s}^{-1}$ (FWHM), roughly consistent with the observed line widths of the ${ }^{13} \mathrm{CO}(2-1)$ and CI emission, are also listed for each model, along with the observed line intensities. The last column in Table 1 gives the reduced $\chi^{2}$ per degree of freedom for each model, $\chi^{2}=\sum(1-$ $\left.I_{\text {mod }} / I_{\text {obs }}\right)^{2} / \sigma_{\text {obs }}^{2} / N$, where $I_{\text {obs }}$ and $I_{\text {mod }}$ are the observed and model line intensities integrated over all velocities, $\sigma_{\text {obs }}$ is the uncertainty of the observed intensities (assumed to be $20 \%$ for all transitions) and $N$ is the number of grid points. The transitions included in the fit are $\mathrm{CO}(2-1), \mathrm{CO}(4-3), \mathrm{CO}(6-5)$, ${ }^{13} \mathrm{CO}(2-1), \mathrm{C}^{18} \mathrm{O}(2-1)$, and $\mathrm{CI}(1-0)$. CO (6-5) has been observed at a position 27" away of the CI peak (position A) (M. Gerin, private communication; observed using the $\mathrm{CSO}$ ) and we assume that the $\mathrm{CO}(4-3) /(6-5)$ ratio there is the same as at the CI peak. $\mathrm{CO}(6-5)$ has not been observed toward positions B and $\mathrm{C}$, and is thus not included in the fit at this location.

The observed line intensities at position A are well reproduced by model $A 1$, with $G_{0}=25, n_{\mathrm{H}}=5 \times 10^{4} \mathrm{~cm}^{-3}$, and $A_{\mathrm{V}}=36$. The extinction is tightly constrained to fit the $\mathrm{C}^{18} \mathrm{O}$ line intensity. With the uncertainties of the dust emission 
Table 1. PDR models of the Horsehead nebula.

\begin{tabular}{|c|c|c|c|c|c|c|c|c|c|c|}
\hline \multicolumn{11}{|l|}{ 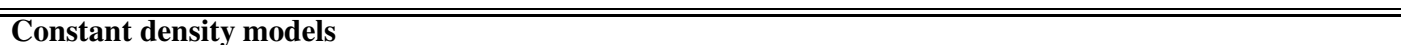 } \\
\hline Model & $n_{\mathrm{H}}$ & $G_{0}$ & $A_{\mathrm{V}}$ & $\begin{array}{c}\mathrm{CO} \\
(2-1)\end{array}$ & $\begin{array}{c}\mathrm{CO} \\
(4-3)\end{array}$ & $\begin{array}{c}\mathrm{CO} \\
(6-5)\end{array}$ & $\begin{array}{l}{ }^{13} \mathrm{CO} \\
(2-1)\end{array}$ & $\begin{array}{l}\mathrm{C}^{18} \mathrm{O} \\
(2-1)\end{array}$ & $\underset{(1-0)}{\mathrm{CI}}$ & $\chi^{2}$ \\
\hline Pos A & & & & 40.5 & 24.8 & 12.0 & 15.2 & 7.3 & 11.1 & \\
\hline $\mathrm{A} 1 *$ & $5 \times 10^{4}$ & 25 & 36 & 43.2 & 29.7 & 8.9 & 14.9 & 7.8 & 11.6 & 0.27 \\
\hline $\mathrm{A} 2$ & $3 \times 10^{4}$ & 25 & 36 & 38.9 & 23.8 & 5.1 & 14.7 & 8.1 & 11.7 & 1.11 \\
\hline A3 & $7 \times 10^{4}$ & 25 & 36 & 46.2 & 33.8 & 12.5 & 14.9 & 7.5 & 11.4 & 0.88 \\
\hline A4 & $5 \times 10^{4}$ & 55 & 36 & 45.2 & 31.8 & 10.4 & 16.2 & 8.4 & 12.9 & 0.61 \\
\hline A5 & $5 \times 10^{4}$ & 10 & 36 & 41.5 & 27.9 & 7.7 & 13.5 & 7.0 & 10.1 & 0.39 \\
\hline A6 & $5 \times 10^{4}$ & 25 & 26 & 42.7 & 29.3 & 8.7 & 14.0 & 6.3 & 11.6 & 0.33 \\
\hline Pos B & & & & 32.5 & 18.2 & - & 9.5 & 0.78 & 5.1 & \\
\hline $\mathrm{B} 1 *$ & $3 \times 10^{4}$ & 1 & 6 & 30.9 & 17.2 & 2.0 & 8.0 & 0.92 & 5.4 & 0.32 \\
\hline $\mathrm{B} 2$ & $3 \times 10^{4}$ & 2 & 6 & 32.7 & 19.0 & 2.6 & 8.1 & 0.56 & 7.1 & 1.29 \\
\hline Pos C & & & & 40.2 & 25.0 & - & 9.20 & 0.89 & 8.5 & \\
\hline $\mathrm{C} 1 *$ & $5 \times 10^{4}$ & 7 & 8 & 38.8 & 26.5 & 7.1 & 9.5 & 0.93 & 9.2 & 0.12 \\
\hline Pos D & & & & 51.7 & 37.8 & 22.7 & 13.8 & 6.0 & 7.4 & \\
\hline $\mathrm{D} 1 *$ & $7 \times 10^{4}$ & Star & 22 & 54.7 & 42.9 & 19.6 & 16.1 & 5.9 & 8.0 & 0.32 \\
\hline \multicolumn{11}{|l|}{ Isobaric models } \\
\hline Model & $p$ & $G_{0}$ & $A_{\mathrm{V}}$ & $\begin{array}{c}\mathrm{CO} \\
(2-1)\end{array}$ & $\begin{array}{c}\mathrm{CO} \\
(4-3)\end{array}$ & $\begin{array}{c}\mathrm{CO} \\
(6-5)\end{array}$ & $\begin{array}{l}{ }^{13} \mathrm{CO} \\
(2-1)\end{array}$ & $\begin{array}{l}\mathrm{C}^{18} \mathrm{O} \\
(2-1)\end{array}$ & $\begin{array}{c}\text { CI } \\
(1-0)\end{array}$ & $\chi^{2}$ \\
\hline D2 & $4 \times 10^{6}$ & Star & 22 & 60.0 & 51.1 & 29.2 & 15.7 & 5.7 & 6.23 & 1.16 \\
\hline D3 & $2 \times 10^{6}$ & & & 54.0 & 42.5 & 20.7 & 15.6 & 5.7 & 6.6 & 0.23 \\
\hline D4 & $1 \times 10^{6}$ & & & 48.4 & 34.7 & 13.7 & 15.4 & 5.8 & 7.2 & 0.77 \\
\hline
\end{tabular}

NoTES: Entries in the table are: Model number, computed intensities of the $\mathrm{CO}(2-1),(4-3),(6-5),{ }^{13} \mathrm{CO}(2-1), \mathrm{C}^{18} \mathrm{O}(2-1)$, and $\mathrm{CI}(1-0)$ emission $\left(\mathrm{K} \mathrm{km} \mathrm{s}^{-1}\right)$, as well as the $\chi^{2}$ value of the fit. The observed line intensities for positions A-C that are used in the fit are also given (we estimate the calibration uncertainty to be $\sim 20 \%$ ). Best-fit models are marked with an asterisk. The radiation field $G_{0}($ Pos $A-C)$ in Draine units. The neck is illuminated double-sided with $G_{0}$ from the front $\underline{\text { and }}$ back side.

coefficients and temperature, the derived extinction is consistent with the $1.2 \mathrm{~mm}$ continuum flux given in the literature $(S=$ $44 \mathrm{mJy}$; D. Tessier priv. comm., Ph.D. Thesis).

Models $A 2$ and $A 3$ show how the output intensities vary with the gas density. High- $J$, means high excitation $\mathrm{CO}$ lines are most affected (compare model $A 2$ vs. $A 3$ and $A 4$ vs. $A 5$, respectively). In particular, the $\mathrm{CO}(6-5) /(2-1)$ ratio increases by a factor of $\sim 2$ for the density increasing from $3 \times 10^{4}$ to $7 \times 10^{4} \mathrm{~cm}^{-3}$. Subthermally excited, high- $J$ CO lines are thus excellent density tracers in PDRs (the density effect is even stronger for the high- $J$ lines of optically thin $\mathrm{CO}$ isotopologues). On the other hand, line intensities of the low-J transitions of the $\mathrm{CO}$ isotopologues, as well as the $\mathrm{CI}$ line are little affected by the gas density. Models $A 4$ and $A 5$ show the effect of $G_{0}$ on the model line intensities. All line intensities increase with $G_{0}$, with CI and CO (6-5) being most affected. Finally, model $A 6$ shows the effect of $A_{\mathrm{V}}$ on the computed line intensities. Only the $\mathrm{C}^{18} \mathrm{O}$ intensity is affected, increasing with $A_{\mathrm{V}}$. All the line intensities in Table 1 are computed assuming a $0^{\circ}$ inclination angle between the line of sight and the line normal to the PDR surface. Increasing the inclination angle affects primarily intensities of the optically thin lines, namely $\mathrm{CI}$ and $\mathrm{C}^{18} \mathrm{O}$. As is clear from this comparison, the different model parameters affect the computed line intensities of different transitions and a good fit can be generally obtained for different locations in the Horsehead.

The observed line intensities at position $B$ are relatively well reproduced by model $B I$ with a hydrogen density of $3 \times$ $10^{4} \mathrm{~cm}^{-3}$, somewhat lower than that derived at position $\mathrm{A}$, and a low value of $G_{0} \simeq 1$. The low CI line intensity is a direct consequence of the low $G_{0}$ value. As illustrated by model $B 2$, increasing the value of $G_{0}$ to 2 results in a CI intensity $\gtrsim 40 \%$ higher than the observed value. Therefore, this part of the neck appears to be well shielded from the FUV photons.
Model $C l$ gives the best fit to the observed line intensities in the mouth area, position $\mathrm{C}$. The derived gas density at this location, $5 \times 10^{4} \mathrm{~cm}^{-3}$ is the same as at the CI peak in the neck (position $\mathrm{A}$ ), and $G_{0} \simeq 7$ is intermediate between the value at positions $\mathrm{A}$ and $\mathrm{B}$. This suggests that the mouth is also partially shielded from the FUV photons, although not as well as the back of the neck. This region may be partially illuminated by the radiation from $\zeta$ Ori to the north.

The volume density and visual extinction values derived from the PDR modeling at positions A and B correspond to the line-of-sight depths of $\sim 230$ and 70", respectively, assuming a distance of $400 \mathrm{pc}$ (Lada et al. 1991). These values are comparable to the width of the nebula in the plane of the sky, confirming our initial assumption of a roughly cylindrical geometry of the neck.

Interestingly, a good fit to the observed line intensities assuming a face-on geometry can also be obtained at position $\mathrm{D}$, on the top of the head (model DI). In this case a somewhat higher gas density of $7 \times 10^{4} \mathrm{~cm}^{-3}$ is required and the FUV illumination assumed in this model is that of an $\mathrm{O} 9.5 \mathrm{~V}$ star at a distance of $4 \mathrm{pc}$, the parameters corresponding to those of $\sigma$ Ori (see Fig. 1 of Pound et al. 2003). The angular resolution of our data is insufficient to resolve the PDR layer in its fine details, but the density we derive from our excitation model for the shielded region is comparable to the density of $\sim 1 \times 10^{5} \mathrm{~cm}^{-3}$ derived by Habart et al. (2005) from their high-resolution IR-spectroscopy of the $\mathrm{H}_{2}$ fluorescent emission and $\mathrm{CO}$ lines.

PDR models $A 3$ and $D 1$ predict face-on CII $158 \mu \mathrm{m}$ line intensities of $1 \times 10^{-4}$ and $2 \times 10^{-4} \mathrm{erg} \mathrm{cm}^{-2} \mathrm{~s}^{-1} \mathrm{sr}^{-1}$, respectively. These are in reasonably good agreement with the observed values of the head (see Fig. 3 of Zhou et al. 1993). The low spatial $\left(55^{\prime \prime}\right)$ and spectral $\left(50 \mathrm{~km} \mathrm{~s}^{-1}\right)$ resolution of the CII data make a more quantitative comparison difficult. Future missions 


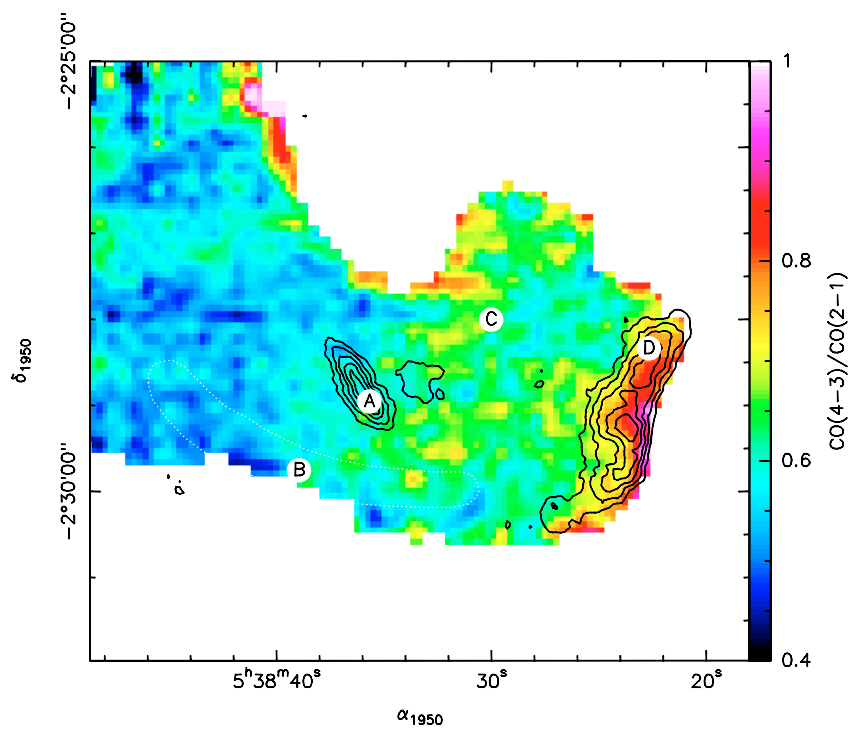

Fig. 6. $\mathrm{CO}(4-3) / \mathrm{CO}(2-1)$ ratio map at $16^{\prime \prime}$ angular resolution. The increased ratio at the western and northern edge of the nebula, indicating enhanced $\mathrm{CO}$ excitation temperatures, shows the direction of incoming UV photons. Symbols and $350 \mu \mathrm{m}$ contours as in Fig. 3 .

(like SOFIA and Herschel/HIFI) will provide the complementary data required for a more detailed analysis.

\subsection{Isobaric models}

All the models presented above assume a constant density distribution throughout the cloud. Given the strong temperature gradient, this assumption results in the gas pressure increasing rapidly toward the surface. Habart et al. (2005) presented a highresolution study of the Horsehead nebula and based on their PDR modeling concluded that the interface region is well described by a constant pressure model, with $p \sim 4 \times 10^{6} \mathrm{~K} \mathrm{~cm}^{-3}$. We therefore run a series of isobaric models equivalent to our model D1 using the Meudon PDR code. The results are shown in Table 1, models D2-D4.

The intensity of the CO (6-5) line is most sensitive to the value of the pressure in the isobaric models and a good fit to the observed line intensities is obtained for $p \sim 2 \times 10^{6} \mathrm{~K} \mathrm{~cm}^{-3}$. This is within a factor of 2 from the value derived by Habart et al. (2005). Given all the modeling and calibration uncertainties, this can be considered as good agreement. We note that the value of $A_{\mathrm{V}}$ is the same in our best fit constant-density and constantpressure models. The resulting column density and mass estimates are thus not affected.

\section{Illumination of the nebula}

Figure 6 shows the $\mathrm{CO}(4-3) /(2-1)$ intensity ratio at $16^{\prime \prime}$ angular resolution. The ratio is relatively uniform across the nebula, with a marked increase at the northern (red rim, Fig. 6) and western edge. Figure 7 shows edge-on peak line intensities of various $\mathrm{CO}$ transitions as a function of distance from the ionization front, computed from PDR model D1 for a $120^{\prime \prime}$ line-of-sight depth. The line intensities are computed from the molecular level population given by the PDR model that are assumed to be constant along each line of sight and to vary only with the distance from the ionization front. The gas kinetic temperature is also shown, along with the $\mathrm{CO}(4-3) /(2-1)$ line ratio (double line, right axis). The CO (4-3)/(2-1) ratio in the model increases toward the edge of the PDR owing to the difference in optical depth

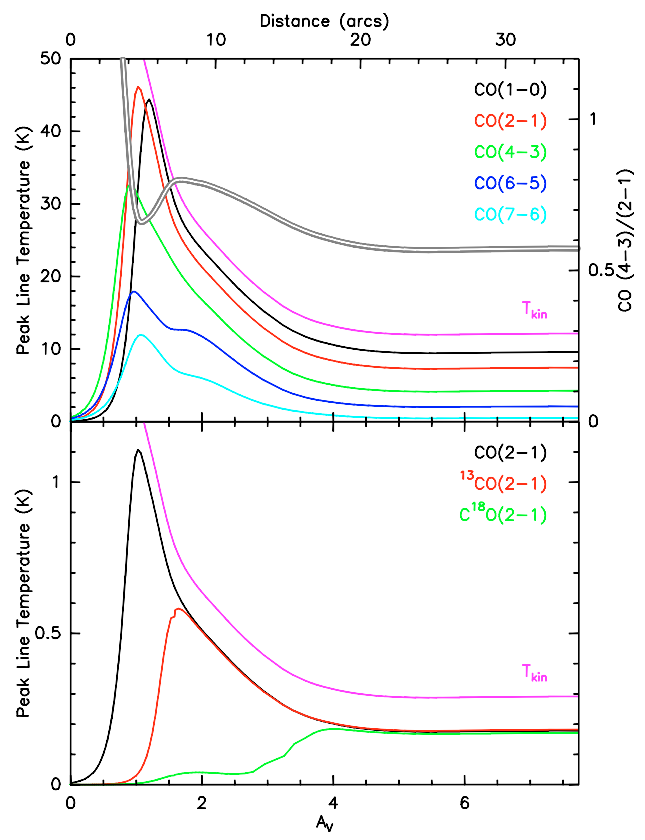

Fig. 7. Peak line intensities of various $\mathrm{CO}$ transitions in the PDR model $D 1$ as a function of distance from the ionization front (assuming a line-of-sight depth of $\left.120^{\prime \prime}\right)$. The $\mathrm{CO}(4-3) /(2-1)$ ratio is shown as a double line (right axis). Ratios of order unity, corresponding to the maximum observed value in Fig. 6, are only present in the outer $A_{\mathrm{V}} \sim 1$ layer. The FWHM telescope beam size of $16^{\prime \prime}$ corresponds to an $A_{\mathrm{V}}$ of $\sim 3.5$.

between the two transitions in the region with a steep temperature gradient. This ratio is thus an excellent tracer of the direction of incoming UV photons. In the case of the Horsehead, the illumination comes primarily from the west ( $\sigma$ Ori), as suggested by the sharp increase of the $\mathrm{CO}(4-3) /(2-1)$ ratio, as well as the bright $\mathrm{H} 2$ fluorescent emission and $\mathrm{PAH}$ emission (Habbart et al. (2005). However the observed increase in the CO (4-3)/(2-1) ratio on the north side of the neck may suggest additional illumination from $\zeta$ Ori $^{1}$ at a projected distance of $30^{\prime}$ to the north. The back of the neck, on the other hand, appears well shielded from the UV photons. This conclusion is consistent with the results of our PDR modeling (Sect. 4).

\section{Mass of the nebula}

The main result of the PDR modeling described above, in addition to constraining the gas density, is the determination of the kinetic temperature and $\mathrm{C}^{18} \mathrm{O}$ and Carbon abundance in the shielded region. With this information, we can accurately determine the molecular mass of the cloud. From the best fit model for the $\mathrm{CI}$ peak in the neck, $A 1$, we derive a fractional $\mathrm{C}^{18} \mathrm{O}$ abundance (with respect to $\mathrm{H}$ nuclei) of $2.4 \times 10^{-7}$ and a kinetic temperature of $\sim 12 \mathrm{~K}$ in the shielded region (Fig. 8). This $\mathrm{C}^{18} \mathrm{O}$ fractional abundance can be compared with the value of $3.3 \times 10^{-7}$ and $1.1 \times 10^{-7}$ derived by Teyssier et al. (2004) and Pety et al. (2005) ("CO-peak" and "Cloud" positions, respectively). Based on LVG models with an $\mathrm{H}$ nuclei density of $5 \times 10^{4} \mathrm{~cm}^{-3}$ and a kinetic temperature of $12 \mathrm{~K}$, we derive a conversion factor from

\footnotetext{
1 The spectral type of $\zeta$ Ori is $\mathrm{O} 9.5 \mathrm{Ib}$, as compared to $\mathrm{O} 9.5 \mathrm{~V}$ for $\sigma$ Ori. The two stars are at roughly the same projected distance from the Horsehead. Depending on the location along the line of sight and the amount of shielding by the protruding material between the star and the nebula (see Fig. 1 of Pound et al.), the illumination from $\zeta$ Ori may be important.
} 


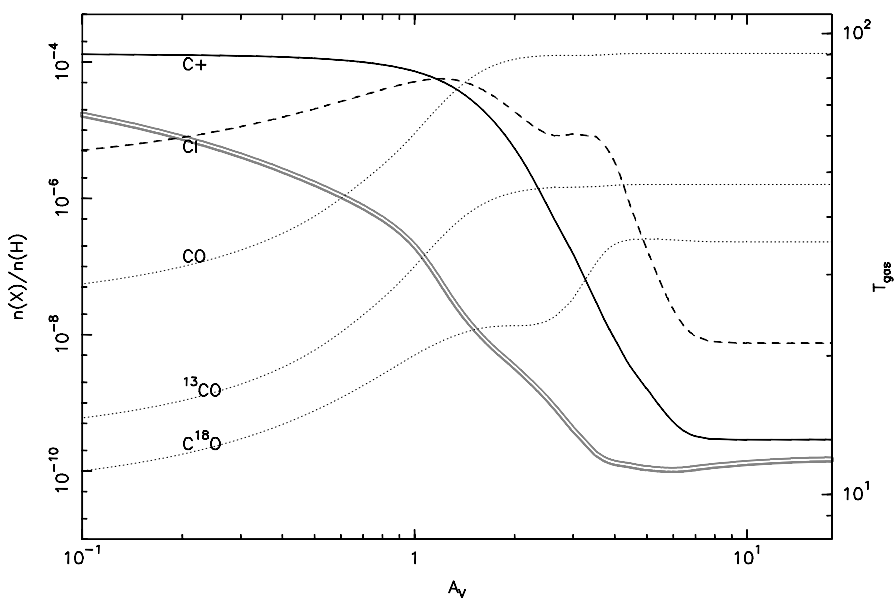

Fig. 8. Temperature structure (double line, right scale) and abundance profiles as a function of $A_{\mathrm{V}}$ for model $A 1$.

the $\mathrm{C}^{18} \mathrm{O}(2-1)$ integrated line intensity to the $\mathrm{C}^{18} \mathrm{O}$ column density of $\sim 7 \times 10^{14} \mathrm{~cm}^{-2}\left(\mathrm{~K} \mathrm{~km} \mathrm{~s}^{-1}\right)^{-1}$. This conversion factor is not sensitive to the volume density and varies by $\lesssim 17 \%$ for kinetic temperatures between 10 and $15 \mathrm{~K}$. Under these assumptions, and using a distance of $400 \mathrm{pc}$, we derive the total mass contained in the $\mathrm{C}^{18} \mathrm{O}$ filament to be $24 M_{\odot}$, slightly lower than the value derived by Pound et al. (2003) based on their CO (1-0) interferometric observations $\left(27 M_{\odot}\right)$.

As seen in Fig. 8, $\mathrm{C}^{18} \mathrm{O}$ is photo-dissociated in the outer PDR layers with $A_{\mathrm{V}} \lesssim 3$, hence does not trace these outer layers. However, the $\mathrm{CI}$ emission is an excellent tracer of this region. In fact, the $\mathrm{C}$ abundance drops just as the $\mathrm{C}^{18} \mathrm{O}$ abundance increases and the two tracers can thus be considered complementary. The $\mathrm{C}$ fractional abundance, with respect to $\mathrm{H}$ nuclei, in the outer envelope reaches a maximum of $5 \times 10^{-5}$ at an $A_{\mathrm{V}} \simeq 1$ and decreases to $1 \times 10^{-5}$ at an $A_{\mathrm{V}} \simeq 2$. The gas kinetic temperature in this region is between 45 and $15 \mathrm{~K}$. The atomic carbon column density can be computed from the LTE formula

$N(\mathrm{C})=1.9 \times 10^{15} \mathrm{~cm}^{-2} \int Q(t) \mathrm{e}^{E_{1} / k T} T_{\mathrm{MB}} \mathrm{d} v$,

where $Q(T)=1+3 \mathrm{e}^{-E_{1} / k T}+5 \mathrm{e}^{-E_{2} / k T}$ is the partition function, and the carbon atom level energies are $E_{1}=23.6 \mathrm{~K}$, and $E_{2}=62.5 \mathrm{~K}$ (e.g. Gerin et al. 1998). For kinetic temperatures in the range $15-45 \mathrm{~K}$, the ratio of the $\mathrm{C}$ column density to the integrated line intensity is approximately $1.4 \times 10^{16} \mathrm{~cm}^{-2}\left(\mathrm{~K} \mathrm{~km} \mathrm{~s}^{-1}\right)^{-1}$. Taking an average $\mathrm{C}$ abundance of $3 \times 10^{-5}$, we derive a mass of the envelope, as traced by the $\mathrm{CI}$ emission to be $\sim 13 M_{\odot}$, about half of the mass in the $\mathrm{C}^{18} \mathrm{O}$ filament. Since $\mathrm{CI}$ and $\mathrm{C}^{18} \mathrm{O}$ emission come from physically distinct regions, we can estimate the total mass of the Horsehead nebula by adding up the two components. The resulting total mass of the cloud is thus $\sim 37 M_{\odot}, \sim 35 \%$ higher than that derived by Pound et al. (2003) based on their CO (1-0) observations alone and in good agreement with the estimate of Lada et al. (1991; $35 M_{\odot}$ ). However, our estimate should be considered an upper limit, since the regions traced by the $\mathrm{C}^{18} \mathrm{O}$ and $\mathrm{CI}$ emission may partially overlap.

We derive a peak CI column density of $\sim 7 \times 10^{16} \mathrm{~cm}^{-2}$ toward the high velocity $\mathrm{CO}$ clump south of the neck (Fig. 1), corresponding to an $\mathrm{H}$ nuclei column density of $2.3 \times 10^{21} \mathrm{~cm}^{-2}$. No $\mathrm{C}^{18} \mathrm{O}$ emission is detected at this location, confirming that this is a low column density fragment poorly shielded from the UV field. The total molecular mass of this clump, based on the CI emission, is only $\sim 0.2 M_{\odot}$.

\section{Summary}

The $\mathrm{CI}$ and $\mathrm{CO}$ isotopologue line intensities observed toward the Horsehead nebula are consistent with PDR models assuming FUV illumination primarily from $\sigma$ Orionis. Our models indicate an average density of $\mathrm{H}$ nuclei of $\sim 3$ (shielded neck) to $7 \times 10^{4} \mathrm{~cm}^{-3}$ (top of the head). The models predict a kinetic temperature of $\sim 12 \mathrm{~K}$ and a $\mathrm{C}^{18} \mathrm{O}$ fractional abundance with respect to $\mathrm{H}$ of $2.4 \times 10^{-7}$ in the shielded region traced by the $\mathrm{C}^{18} \mathrm{O}$ emission. Towards the PDR layers facing the ionizing star(s), temperatures increase to $\sim 50 \mathrm{~K}$ at an $A_{\mathrm{V}}$ of 1 (nicely reflected in the enhanced $\mathrm{CO}(4-3) /(2-1)$ line ratio). Using these parameters, we derive a total molecular mass of $24 M_{\odot}$ in the $\mathrm{C}^{18} \mathrm{O}$ filament. The outer halo devoid of $\mathrm{C}^{18} \mathrm{O}$ emission, but traced by the CI emission contributes additional $13 M_{\odot}$ of material, leading to upper limit of $\sim 37 M_{\odot}$ for the total mass of the nebula.

Acknowledgements. This research has been supported by NSF grant AST 2209008 to the Caltech Submillimeter Observatory. We thank D. Dowell for providing us with the $350 \mu \mathrm{m}$ SHARC II image prior to publication and J. Bally for the mosaic on the horsehead of the NOAO Star Formation Survey Data 1997 in $\mathrm{H} \alpha$ emission, J. Le Bourlot and E. Roueff for their assistance with the Meudon PDR code, and M. Gerin, K. M. Menten, P. Schilke and M. Walmsley for stimulating discussions. Also we like to thank the CSO staff for their help and support during the CHAMP campaigns.

\section{References}

Abergel, A., Teyssier, D., Bernard, J. P., et al. 2002, A\&A, 410, 577 Abergel, A., Bernard, J. P., Boulanger, F., et al. 2003, A\&A, 389, 239 Abgrall, H., Le Bourlot, J., Pineau des Forêts, G., et al. 1992, A\&A, 253, 525 Fitzpatrick, E. L., \& Massa, D. 1990, ApJS, 72, 163

Gerin, M., Phillips, T. G., Keene, J., Betz, A. L., \& Boreiko, R. T. 1998, ApJ, 500,329

Güsten, R., Ediss, G. A., Gueth, F., et al. 1998, in Advanced Technology MMW, Radio and Terahertz Telescopes, Proc. SPIE, 3357, 167, ed. T. G. Phillips, Kona

Habart, E., Abergel, A., Walmsley, C. M., Teyssier, D., \& Pety, J. 2005, A\&A, 437, 177

Habing, H. J. 1968, Bull. Astron. Inst. Netherlands, 19, 421

Hollenbach, D. J., \& Tielens, A. G. G. M. 1997, ARA\&A, 35, 179

Hollenbach, D. J., \& Tielens, A. G. G. M. 1999, Rev. Mod. Phys., 71, 173

Keene, J., Lis, D. C., Phillips, T. G., \& Schilke, P. 1997, in Molecules in Astrophysics: Probes and Processes, ed. E. van Dishoeck (Kluwer: Dordrecht), 129

Kramer, C., Stutzki, J., \& Winnewisser, G. 1996, A\&A, 307, 915

Lada, E. A., Bally, J., \& Stark, A. A. 1991, ApJ, 368, 432

Le Bourlot, J. 2000, A\&A, 360, 656

Le Bourlot, J., Pineau des Forêts, G., Roueff, E., \& Flower, D. 1993, A\&A, 267, 233

Le Petit, F., Nehmé, C., Le Bourlot, J., \& Roueff, E. 2006, ApJS, in press

Lis, D. C., Schilke, P., \& Keene, J. 1997, in CO: Twenty-Five Years of Millimeter-Wave Spectroscopy, ed. W. Latter et al. (Dordrecht: Kluwer), 128

Pety, J., Teyssier, D., Fossé, D., Gerin, M., et al. 2005, A\&A, 435, 885

Pound, M., Reipurth, B., \& Bally, J. 2003, AJ, 125, 2108

Reipurth, B., \& Bouchet, P. 1984, A\&A, 137, L1

Sandell, G., Reipurth, B., Menten, K., Walmsley, C. M., \& Ungerechts, H. 1985, in Light on Dark Matter, ed. F. P. Israel (Dordrecht: Reidel), 295

Schuster, K., Greve, A., Hily-Balnt, P., et al. 2002, IRAM manual, available at http://www.iram.es/IRAMES/other Documents/manuals/ HERA_manual_v15.pdf

Seaton, M. J. 1979, MNRAS, 187, 73P

Sternberg, A. 2005, in Astrochemistry: Recent Successess and Current Challenges, ed. D. Lis, G. Blake, \& E. Herbst (CUP), 141

Teyssier, D., Fossé, D., Gerin, M., et al. 2004, A\&A, 417, 135

Yamauchi, S., Kamimura, R., \& Koyama, K. 2000, PASJ, 52, 1087

Zhou, S., Jaffe, D. T., Howe, J. E., et al. 1994, ApJ, 419, 190 